\title{
EXTENDED DEFECTS AND POLARITY OF HYDRIDE VAPOR PHASE EPITAXY GaN
}

\author{
J. Jasinski ${ }^{\text {a) }}$ and Z. Liliental-Weber \\ Lawrence Berkeley National Laboratory, 1 Cyclotron Road, Berkeley, CA 94720
}

Hydride vapor phase epitaxy (HVPE) GaN layers on sapphire substrates and so-called free-standing platelets (layers removed from the sapphire) were studied by different transmission electron microscopy (TEM) techniques. Polarity determined by convergent beam electron diffraction (CBED) and distribution of structural defects, determined by conventional TEM, are discussed. HVPE layers were found to grow primarily with Gapolarity. A few inversion domains (areas with N-polarity) were observed on the substrate side of one of the free-standing layers. The dominant structural defects in HVPE GaN layers are threading dislocations. A systematic reduction of their density with increase in layer thickness was observed for all samples. The experimental results indicate that the density of dislocations is inversely proportional to the distance from the substrate, which agrees with the theoretical model.

a) Also with: the Institute of Experimental Physics, Warsaw University, Hoza 69, 00-681 Warsaw, Poland 


\section{INTRODUCTION}

In recent years, GaN-based semiconductors have attracted scientific interest because of their importance in many applications, mostly in optoelectronic, and highpower, and high-temperature electronic devices. Good quality GaN-based light-emitting diodes, blue lasers and metal-semiconductor field-effect transistors have been demonstrated. ${ }^{1-6}$ Despite the large number of applications and wide spread interest in GaN and other III-nitrides there are still some problems associated with the technology of these materials. One of them is the problem with available substrates for epitaxial growth of nitride semiconductor layers. There are still some difficulties in growing large bulk GaN crystals ${ }^{7}$, mostly due to the high $\mathrm{N}$ overpressure on GaN and the very small solubility of $\mathrm{N}$ in a Ga melt. Therefore, production of large area GaN crystals has not yet been realized. Instead, nitride layers have been grown on many foreign substrates, including $\mathrm{ZnO}, \mathrm{MgAl}_{2} \mathrm{O}_{4}, \mathrm{Si}, \mathrm{GaAs}, \mathrm{MgO}, \mathrm{NaCl}, \mathrm{W}$ and $\mathrm{TiO}_{2} . \mathrm{SiC}^{8}$, and especially sapphire $^{9-12}$ are most commonly used. Large differences in lattice parameter and in thermal expansion coefficient between $\mathrm{GaN}$ and sapphire result in a high density of threading dislocations. ${ }^{13-14}$ There are different approaches for overcoming this problem and reducing the number of dislocations. Since GaN films grown on sapphire consist of many slightly twisted/tilted subgrains separated by small angle grain boundaries formed by the threading dislocations some methods have focused on grain alignment or in the case of lateral epitaxial overgrowth on increasing grain size. ${ }^{15}$ Other methods utilize the presence of intermediate layers where interactions between dislocations are enhanced so that they can combine or annihilate. ${ }^{16-17}$ Finally, since the density of threading dislocations approaching the layer surface decreases with increase of the layer thickness, 
an alternative method of obtaining GaN films with a low density of threading dislocations is homoepitaxial growth on very thick GaN layers on sapphire or other substrates. Consequently, attention has turned to the growth of very thick GaN films by hydride vapor-phase epitaxy (HVPE). ${ }^{18-21}$ However, in order to efficiently use this method and in a controlled way reduce the density of dislocations a good understanding of the mechanism responsible for such reduction is needed. There are only a few papers in the literature, which discus the dislocation reduction process in thick HVPE GaN layers and their conclusions seemed to be somewhat contradictory. Based on experimental data and some theoretical considerations Albrecht et al. concluded that very efficient reduction of dislocation density takes place when only pure edge dislocations are present in the layer and that a small density reduction occurs when dislocations are distributed with all Burgers vectors. ${ }^{22}$ On the contrary, Mathis et al. presented a theoretical model which leads to the conclusions that films with a high fraction of edge dislocations do not have significant dislocation reduction and mixed character dislocations promote the reduction reactions. ${ }^{23}$ It seems than, that there is still some controversy about the dislocation density reduction mechanism and some additional experimental or/and theoretical studies are necessary. We believe that results of systematic studies presented in this paper can be an important experimental contribution to the discussion about the mechanism of dislocation reduction in HVPE GaN layers.

In this paper we present and discuss the results of structural studies, performed by the use of transmission electron microscopy (TEM) on a series of HVPE GaN layers grown on sapphire. We examined two types of layers: layers attached to the substrate and so-called free-standing layers, e.g. layers separated from the substrate. Since there is little 
known about the polarity of HVPE GaN layers the first part of this paper will present results of convergent beam electron diffraction (CBED) studies and discuss polarity in this material. The second, part will describe the nature and distribution of extended defects present in these layers, among which threading dislocations are dominant. In particular, we will present quantitative experimental results showing reduction of threading dislocation density with increase of layer thickness and will analyze this data to compare with available published experimental data and theoretical models.

\section{EXPERIMENT}

HVPE GaN layers grown by two different groups were used for this study. Four layers were grown with nominal thicknesses of $1.5 \mu \mathrm{m}, 2.5 \mu \mathrm{m}, 5.5 \mu \mathrm{m}$ and $55 \mu \mathrm{m}$ on sapphire at the MIT Lincoln Laboratory (for details of growth conditions see Ref. 24) and two free-standing layers of a thickness about $200 \mu \mathrm{m}$ were grown at the Samsung Advanced Institute of Technology. The last two samples (they will be referred to later in this paper as samples: FS1 and FS2) were also grown on a sapphire substrate and their initial thickness before laser induced lift off ${ }^{25}$ was about $300 \mu \mathrm{m}$. After separation from the substrate these layers were mechanically polished, and dry etched on their original surface to obtain a smooth epi-ready surface. The opposite surface was only mechanochemically polished. For these two layers TEM plan-view specimens were prepared from both sides and from the remaining ones with the exception of the sample with the thickness of $55 \mu \mathrm{m}$ where the large accumulated stress caused constant breaking only from the surface side. All TEM specimens were prepared by a standard method of mechanical pre-thinning followed by Ar-ion milling down to electron transparency. TEM 
studies were carried out using a TOPCON 002B microscope, operated at $200 \mathrm{kV}$ acceleration voltage.

\section{RESULTS AND DISCUSSION}

\section{CRYSTAL POLARITY}

The [0001] direction of the GaN layer grown on a c-plane sapphire substrate can have two different polarities. These polarities correspond to orientation of the Ga-N bond, which is parallel to the c-axis. A layer has a Ga-polarity when the growth direction points from the $\mathrm{Ga}$ atom towards the $\mathrm{N}$ atom. When the growth direction points from the $\mathrm{N}$ atom towards the Ga atom a layer has a N-polarity.

In order to determine the polarity of the investigated $\mathrm{GaN}$ layers convergent beam

electron diffraction (CBED) studies were applied. ${ }^{26-28}$ Since GaN is a non-centrosymetric crystal, the intensity distribution within " $+\mathrm{g}$ " and "-g" diffraction discs in the CBED pattern are different and this difference can be attributed to $\mathrm{Ga}$ and $\mathrm{N}$ distribution within the unit cell. In our studies of cross-sectional specimens we have chosen the [1100] zone axis CBED pattern for two reasons: one - it contains the (0002) and (0002) diffraction discs with different intensity distribution due to crystal polarity and second - it contains a small number of well separated discs and therefore it is easily interpretable. Since the intensity distribution within the CBED discs depends also on sample thickness, the central - (0000) - disc was first used to determine the sample thickness and then a CBED pattern was simulated for this specific thickness to match asymmetrical intensity distribution within the (0002) and (0002) diffraction discs. To assure the correctness of polarity assignment for each layer [1 100$]$ zone axis CBED patterns were recorded in 
different regions, corresponding to several TEM specimen thicknesses and matched with simulated patterns.

The method described above was applied to all layers attached to sapphire substrates and it was found that they grow with Ga polarity. An example of an experimental CBED pattern recorded for the HVPE layer with a thickness of $2.5 \mu \mathrm{m}$ is shown in Fig. 1 for the TEM sample thickness of $210 \mathrm{~nm}$. A very good match between the experimental and calculated pattern for this sample thickness was obtained.

It is well documented in the literature that under certain conditions inversion domains (ID), e.g. regions with opposite polarity, can be formed during $\mathrm{MOCVD}^{29-31}$ or $\mathrm{MBE}^{32}$ growth of GaN layers. There are also some indications that such domains can be formed in GaN during HVPE growth. ${ }^{33}$ Taking this into consideration, for each layer we checked polarity in many different areas of the TEM samples and only one, Ga-polarity was found. There were no IDs observed in the layers attached to the sapphire substrate (e.g. grown at MIT).

For both free-standing layers CBED studies were performed on both sides of the crystals, that means close to the original surface and close to the rough surface formed during layer lift-off. In case of sample FS1 these studies showed that the layer has Gapolarity. As in the case of layers attached to sapphire no IDs were observed. Sample FS2 was found to have predominately Ga-polarity. However, a relatively high density of IDs was observed close to the substrate side. Their presence was first indicated by diffraction contrast studies showing that ID boundaries disappear for g-vector perpendicular to the caxis of the GaN crystal and are in strong contrast for the g-vector parallel to the c-axis (see Fig. 2). ${ }^{29,30}$ The presence of these IDs was confirmed by CBED study showing N- 
polarity within such domains. Fig. 3 (a) shows a TEM micrograph of the area on the substrate side with some IDs. The CBED pattern recorded from within such an ID with the simulated pattern and a schematic distribution of atoms along the growth direction are shown in Fig. 3(b). Analogous patterns and schematic distributions of atoms for a neighboring, defect-free area are shown in Fig. 3(c). One can notice that, again a very good match between experimental and simulated patterns was obtained and that the ID has N-polarity, opposite to that of the surrounding material. The formation mechanism of such IDs during growth of some HVPE GaN layers is not yet clear and its understanding will require future studies.

\section{EXTENDED DEFECTS}

\section{Misfit dislocations}

The large lattice mismatch (about $14 \%$ ) between GaN and sapphire results in a large number of misfit dislocations at the interface between these two materials which are the result of plastic deformation that relieves the large strain accumulated in the system. The number of misfit dislocations present at the interface can be estimated based on high resolution electron microscopy (HREM) images. We recorded such images for layers attached to the sapphire substrate and filtered them in Fourier space so the misfit dislocations present at the interface were clearly visible. From an analysis of such filtered HREM images we estimated the average distance between misfit dislocations at the interface to be about $20 \AA$. Similar values for the GaN/sapphire interface have been reported in the literature. ${ }^{34}$ 


\section{Threading Dislocations}

Lattice mismatch and difference in thermal expansion coefficients between GaN and sapphire also result in the formation of a high density of threading dislocations in GaN layers grown on sapphire. Threading dislocations were found to be the dominant extended defects in all our investigated HVPE layers (except for sample FS2 where the relatively high density of IDs was observed at the substrate side). Threading dislocations were observed in both, cross-sectional and plan-view images. Examples of such images recorded for the $2.5 \mu \mathrm{m}$ thick layer are shown in Figs 4(a) and 4(b), respectively. A majority of these threading dislocations were found to propagate through the GaN film parallel to the c-axis. In all the layers attached to the sapphire the highest density of dislocations, in the range of $10^{10} \mathrm{~cm}^{-2}$, was observed in the region $0.1-0.2 \mu \mathrm{m}$ from the GaN/sapphire interface. As distance the from the substrate increases the dislocation density decreases due to interactions between dislocations. Consequently for each crosssectional specimen the lowest dislocation density was measured in the vicinity of the top surface. The data from cross-sectional and plan-view samples are in good agreement. Results of dislocation density measurements are summarized in Table I and plotted in Fig. 5 as a function of distance from the substrate. Results obtained from free-standing layers are also included. Their dislocation density was estimated at specific distances from the original interface with the substrate. The original thickness of these layers was about $300 \mu \mathrm{m}$ and after liftoff and mechano-chemical polishing it was reduced to about $200 \mu \mathrm{m}$. In order to include in the plot the dislocation density data, obtained from freestanding layers, the assumption was made that $\mathrm{N}$ - and Ga-surfaces, formed after polishing, were initially at distances of $\sim 100 \mu \mathrm{m}$ and $\sim 300 \mu \mathrm{m}$, respectively, form the 
sapphire substrate. Table I and the plot shown in Fig. 5 also include an experimental point representing the lowest measured dislocation density obtained on HVPE GaN grown on sapphire. ${ }^{35}$ It was achieved on a $750 \mu \mathrm{m}$ thick Samsung layer.

In our studies we also investigated the distribution of threading dislocations among different types of Burgers vectors. It is well known that there are primarily three types of threading dislocations in $\mathrm{GaN}$ films each having one of the three Burgers vectors: $1 / 3<11-20>,<0001>$ and $1 / 3<11-23>$ representing edge, screw and mixed dislocations, respectively with the assumption that the dislocation lines lie parallel to the c-axis. ${ }^{36}$ In order to identify the Burger's vector of a dislocation one needs to record at least two diffraction conditions for which the dislocation contrast disappears, however based on two images (recorded for g-vector parallel and perpendicular to the c-axis) one can identify the type of dislocation. An edge dislocation will appear only in the image recorded for g-vector perpendicular to the c-axis whereas a screw dislocation will be visible only in the image recorded for g-vector parallel to the c-axis. On the other hand, a mixed-type dislocation will appear in both images. In our study we applied such a method to estimate the relative densities of the different types of dislocations. For each layer we recorded pairs of images for both types of g-vectors: parallel and perpendicular to the caxis. An example of such a pair of bright-field images obtained for a $2.5 \mu \mathrm{m}$-thick layer and a pair of dark-field images obtained for the $5.5 \mu \mathrm{m}$-thick layer are shown in Figs 6 and 7, respectively. Such an analysis showed that in the thinner layers $(2.5 \mu \mathrm{m}$-thick) all three types of dislocations were present in similar numbers. For $5.5 \mu \mathrm{m}$-thick layer the numbers of edge and screw dislocations were similar whereas the number of mixed dislocations was about two times higher. Similar experiments performed on the free- 
standing templates, which were much thicker, showed that most of the remaining threading dislocations present in these templates were of mixed Burger's vector. However, due to a very low density of dislocations, there were only a few dislocations visible within the electron transparent area of the TEM specimens and so the level of confidence of such assignment of dislocation type distribution is much lower.

Our studies on distribution of threading dislocations in HVPE GaN layers can be helpful in understanding the dislocation density reduction mechanism in this material. Such reduction process visible from the trend of experimental data presented in Fig. 5 can be qualitatively described as follows: In the early stages of growth, due to the high lattice mismatch between GaN and sapphire, the GaN film starts to grow in an island mode, characterized by many separate nucleation sites. This results in nucleation of many slightly tilted/twisted GaN grains and a high density of threading dislocations, which appear at merging points of neighboring grains. In addition some can also be related to the misfit dislocation network. Once threading dislocations are formed they propagate into the layer during growth. As a consequence for a planar growth surface threading dislocations lie primarily parallel to the growth direction. These dislocations can interact with each other either by combining or annihilation and these interactions are responsible for the reduction in dislocation density. In the vicinity of the GaN/sapphire interface there are many inclined dislocations due to the island growth mode [see for example Fig. 4(a)]. Such dislocations have a much greater chance to meet another dislocation and interact with it. As distance from the substrate increases the number of inclined dislocations decreases compared to the number of dislocations parallel to the c-axis because the roughness of the growth surface decreases. As a result the probability of dislocation 
interactions decreases with an increase of distance from the substrate and the process slows down. Further decrease of dislocation density for thicker layers becomes increasingly difficult since their line directions are primarily parallel to the c-axis and the average separation between them is greater. This reduces significantly the probability of interactions between dislocations.

There have been few attempts in literature to formulate a theory, which describes interactions of threading dislocations and reduction of their density. In one publication Speck et al. ${ }^{37}$ proposed a model according to which, overall density of threading dislocations $\rho$ in relatively thick layers is inversely proportional to the film thickness $\mathrm{h}$ :

$$
\rho=1 / \mathrm{Kh}
$$

where, $\mathrm{K}$ is a dislocation reaction cross-section. The authors used this model successfully to explain the $1 / \mathrm{h}$ - scaling behavior observed experimentally in several cubic materials by Sheldon et al. ${ }^{38}$ We applied this model to our experimental data from HVPE GaN layers presented in Fig. 5. Surprisingly, this model seems to work very well also in this case, as can be seen from Fig. 5 where the curve representing the best fit of equation (1) was shown in addition. This fit was obtained for a value of $308 \pm 35 \AA$ for the $\mathrm{K}$ parameter. Mathis et al. $^{23}$ applied a similar approach to analyze experimental data reported by Golan et al. ${ }^{39}$ obtained on HVPE GaN using plan-view TEM and X-ray diffraction measurements. They obtained a value of $275 \AA$ for K, which agrees well with our value of $308 \pm 35 \AA$ for this parameter.

Mathis et al. $^{23}$ extended the model proposed by Speck et al. ${ }^{37}$ for cubic semiconductors into the case of wurzite GaN. According to this model efficiency and kinetics of dislocation reduction strongly depends on the initial dislocation distribution 
between different types of Burgers vectors. The authors performed several simulations, which show such strong dependence. There is also an experimental paper by Albrecht et al. ${ }^{22}$, which emphasizes the role of initial dislocation population on efficiency of dislocation density reduction. However, these two papers seem to be somewhat contradictory. Mathis et al. ${ }^{23}$ concluded that there is an efficient reduction of threading dislocation density in films with a high fraction of mixed character dislocations since only such dislocations tend to be inclined. On the other hand, films with a high fraction of edge dislocations should not have significant dislocation reduction. Contrary to this statement Albrecht et al. ${ }^{22}$ claimed that only films that have primarily only edge type dislocations exhibit strong dislocation density reduction. Our results obtained on HVPE GaN layers seem to support the first of these two papers. We observe a strong dislocation reduction (by almost four orders of magnitude in films of a thickness of a few hundreds micrometers) and the investigated films had a large fraction of mixed character dislocations. On the other hand, there are still some discrepancies between our experimental data and predictions of the theoretical model presented by Mathies et al. ${ }^{23}$ For example, this model predicts that dislocation density would saturate for large layer thickness and there is no such saturation observed in our data (see Fig. 5). Similarly, the authors of this theoretical paper did not observe such a saturation for experimental data by Golan et $\mathrm{al}^{39}$. We believe however that this theoretical model is correct and that saturation will appear for films thicker than these which were investigated.

\section{SUMMARY}


Transmission electron microcopy (TEM) was applied to study HVPE GaN layers grown on sapphire. CBED studies performed on both, thin, attached to the substrate films, and thick, free-standing layers showed that they grow with Ga polarity. However, in case of one thick free-standing layer a relatively high density of inversion domains having N-polarity were observed near the "substrate side". Their presence was confirmed by both, CBED and diffraction contrast analysis. The origin of these inversion domains is still not clear but since these defects terminated within the layer and did not propagate to the original free surface of the thick layer, they should not effect properties and quality of an epitaxial layer, grown on such a substrate.

A high density of misfit dislocations was present at the substrate/layer interface in the attached layers. The average distance between them was estimated to be about $20 \AA$ as reported in the literature for the $\mathrm{GaN} / \mathrm{Al}_{2} \mathrm{O}_{3}$ system. These misfit dislocations are associated with the threading dislocations, which are the dominant structural defects within the HVPE GaN layers. All three types of threading dislocation (edge, screw and mixed) with similar numbers were observed. For all samples attached to the sapphire the highest density (in the range of $10^{10} \mathrm{~cm}^{-2}$ ) was observed in a thin region adjacent to the GaN/sapphire interface. As the distance from the substrate increased a gradual reduction of threading dislocation density (resulting from interactions between dislocations) was observed. This reduction was well described by the $1 / \mathrm{h}-$ scaling factor predicted by the theoretical models of Speck et al. ${ }^{37}$ (for cubic materials) and Mathis et al. ${ }^{23}$ (for hexagonal GaN layers). Our experimental data showing a high rate of threading dislocation reduction with thickness increase along with the presence of a high fraction of mixed type dislocations supports the theoretical model of dislocation reduction proposed 
by Mathis et al. ${ }^{23}$ This model predicts that the density of dislocation will tend to saturate with increase of layer thickness. Our results suggest that this saturation level should be near or even below the $10^{6} \mathrm{~cm}^{-2}$ value. With this low a density of dislocations, thick GaN layers grown by HVPE should be useful templates for homoepitaxial growth of GaN. Moreover, the fact that the results obtained on layers having different thicknesses and grown in two different laboratories had the same polarity and followed the same trend of dislocation reduction suggests good reproducibility for HVPE growth.

\section{ACKNOWLEDGMENTS}

This work was supported by the Air Force Office of Scientific Research, through the U.S. Department of Energy under Order No. AFOSR-ISSA-00-0011 (Wood-Witt program). The authors would like to thank Dr. R. J. Molnar from the Massachusetts Institute of Technology, and Drs S. S. Park and K. Y. Lee from the Samsung Advanced Institute of Technology for growing and providing HVPE GaN layers, Drs D. Look and H. Morkoç for coordination of this program, W. Swider for excellent TEM sample preparation, and finally Dr. M. Benamara and Prof. J. Washburn for very fruitful discussions. The authors want to acknowledge the use of the facilities at the National Center for Electron Microscopy at Lawrence Berkeley National Laboratory.

\section{REFERENCES}

1. S. Nakamura, M. Senoh, S. Nagahama, N. Iwasa, T. Yamada, T. Matsushita, Y. Sugimoto, H. Kiyoku, Appl. Phys. Lett. 69, 4056 (1996).

2. S. N. Mohammad, A. Salvador and H. Morkoc, Proc. IEEE 83, 1306 (1995). 
3. F. A. Ponce and D. P. Bour, Nature 386, 351 (1997).

4. S. Strite and H. Morkoc, J. Vac. Sci. Technol. B. 10, 1237 (1992).

5. R. F. Davis, Proc. IEEE 79, 702 (1991).

6. H. Morkoc, Nitride Semiconductors and Devices (Springer, Heidelberg, 1999).

7. I. Grzegory, J. Jun, M. Bockowski, S. Krokowski, M. Wroblewski, B. Lucznik and S. Porowski, J. Phys. Chem. Solids 56, 639 (1995).

8. P. Vermaut, R. Ruterana and G. Nouet, Philos. Mag. A 75, 239 (1997).

9. T. Lei, K. F. Ludwig Jr. and T. Moustakas, J. Appl. Phys. 74, 4430 (1993).

10. G. Popovici, W. Kim, A. Botchkarev, H. Tang and H. Morkoc, Appl. Phys. Lett. 71, 3385 (1997).

11. N. P. Kobayashi, J. T. Kobayashi, P. D. Dapkus, W. -J. Choi, A. E. Bond, X. Zhang and D. H. Rich, Appl. Phys. Lett. 71, 3569 (1997).

12. G. Y. Zhang, Y. Z. Tong, Z. J. Yang, S. X. Jin, J. Li and Z. Z. Gan, Appl. Phys. Lett. 71, $3376(1997)$.

13. Z. Liliental-Weber, S. Ruvimov, W. Swider, Y. Kim. J. Washburn, S. Nakamura, R.S. Kern, Y. Chen, and J.W. Yang, Mat. Res. Soc. Proc. 482, 375 (1998).

14. F. Ponce, D.P. Bour, W. Gotz, and P.J. Wright, Appl. Phys. Lett. 68, 57 (1996).

15. O. -H. Nam, M. D. Bremser, T. S. Zheleva and R. F. Davis, Appl. Phys. Lett. 71, 2638 (1997).

16. H. Amano, M. Iwaya, N. Hayashi, T. Kashima, M. Katsuragawa, T. Takeuchi, C. Wetzel, and I. Akasaki, MRS Internet J. Nitride Semicond. Res. 4S1, G10.1 (1999).

17. D. D. Koleske, M. E. Twigg, A. E. Wickenden, R. L. Henry, R. J. Gorman, J. A. Freitas, Jr., and M. Fatemi, Appl. Phys. Lett. 75, 3141 (1999). 
18. S. Nakamura, M. Senoh, S. Nagahama, N. Iwasa, T. Yamada, T. Matsushita, H. Kiyoku, Y. Sugimoto, T. Kozaki, H. Umemoto, M. Sano, and K. Chocho, Appl. Phys. Lett., 73, 832 (1998).

19. S.T. Kim, Y. J. Lee, D. C. Moon, C.H. Hong, T.K. Yoo, J. Cryst. Growth, 194, 37, (1998).

20. O. Kryliouk, M. Reed, M. Mastro, T. Dann, T. Anderson, B. Chai, Proc. of Third Symp. III-V nitride Materials and Processes, Electr. Chem. Soc. p.99 (1999).

21. R. P. Vaudo, VM. Phanse, Proc. of Third Symp. III-V Nitride Materials and Processes, Electr. Chem. Soc. p.79 (1999).

22. M. Albrecht, I.P. Nikitina, A.E. Nikolaev, Yu.V. Melnik, V.A. Dmitriev, H.P. Strunk, phys. stat. sol. (a) 176, 456 (1999).

23. S.K. Mathis, A.E. Romanov, L.F. Chen, G.E. Beltz, W. Pompe, J.S. Speck, phys. stat. sol. (a) 179, 125 (2000).

24. R. J. Molnar, W. Gotz, L. T. Romano, N. M. Johnson, J. Cryst. Growth 178, 147 (1997).

25. M. K. Kelly, R. P. Vaudo, V. M. Phanse, L. Gorgens. O. Ambacher. M. Stutzmann, J. J. Apl. Phys., 38, L217, (1999).

26. Z. Liliental-Weber, C. Kisielowski, S. Ruvimov, Y. Chen, J. Washburn, I. Grzegory, M. Bockowski, J. Jun, S. Porowski, J. Electron. Mat. 25, 1545 (1996).

27. J. -L. Rouviere, M. Arlery, A. Bourret, R. Niebuhr, K. -H. Bachem, Mat. Res. Soc. Symp. Proc. 395, 393 (1996).

28. Z. Liliental-Weber, M. Benamara, O. Richter, W. Swider, J. Washburn, I. Grzegory, S. Porowski, J. W. Yang, S. Nakamura, Mat. Res. Soc. Symp. Proc. 512, 363 (1998). 
29. X. H. Wu, L. M. Brown, D. Kapolnek, S. Keller, B. Keller, S. P. DenBaars, and J. S. Speck, J. Appl. Phys. 80, 3228 (1996).

30. L. T. Romano, J. E. Northrup, and M. A. O'Keefe, Appl. Phys. Lett. 69, 2394 (1996).

31. D. Cherns, W. T. Young and F. A. Ponce, Mater. Sci. and Eng. B50, 76 (1997).

32. V. Potin, P. Ruterana and G. Nouet, Mater. Sci. and Eng. B59, 173 (1999).

33. Chinkyo Kim, Min Yang, Wonsang Lee, Jaehyung Yi, Sungwoo Kim, Yoonho Choi, Tae-Kyung Yoo and Seon Tai Kim, J. Cryst. Growth 213, 235 (2000).

34. S. Kaiser, H. Preis, W. Gebhardt, O. Ambacher, H. Angere, M. Stutzmann, A. Rosenauer, and D. Gerthsen, Jpn. J. Appl. Phys. 37, 84 (1998).

35. K. Lee, K. Auh, MRS Internet Journal, Nitride Semiconductor Research 6, 9 (2001).

36. D. Hull, D.J. Bacon, “Introduction to disolcations”, Pergamon Press, 1984.

37. J. S. Speck, M. A. Brewer, G. Beltz, A. E. Romanov, and W. Pompe, J. Appl. Phys. 80, 3808 (1996).

38. P. Sheldon, K. M. Jones, M. M. Al-Jassim, and B. G. Yacobi, J. Appl. Phys. 63, 5609 (1988).

39. Y. Golan, X. H. Wu, J. S. Speck, R. P. Vaudo, and V. M. Phanse, Appl. Phys. Lett. 73, 3090 (1998). 


\section{TABLE AND FIGURE CAPTIONS}

Table. I. Density of threading dislocations as a function of the distance from the substrate measured from cross-sectional (c-s) and plan-view (p-v) specimens prepared from attached and free-standing HVPE GaN layers. The lowest published value (see Ref. 35) measured for a $750 \mu$ m-thick HVPE GaN layer is added.

Fig. 1. Polarity determination: TEM image of $2.5 \mu \mathrm{m}$-thick layer showing the position of the beam for CBED analysis, experimental and simulated (for $210 \mathrm{~nm}$ ) CBED patterns, and schematic distribution of atoms along the growth directions.

Fig. 2. Two-beam bright-field images of the substrate side of sample FS2 recorded for gvector (a) perpendicular and (b) parallel to the c-axis.

Fig. 3. (a) TEM image of the area containing some IDs on the substrate side of sample FS2. Experimental and simulated CBED patterns, and schematic distribution of atoms along the growth directions obtained (b) on and (c) outside such ID. Simulated CBED patterns were calculated for TEM specimen thickness of $85 \mathrm{~nm}$ and $90 \mathrm{~nm}$, respectively.

Fig. 4. (a) Cross-sectional and (b) plan-view TEM images of a $2.5 \mu \mathrm{m}$-thick GaN HVPE layer. Threading dislocations are the dominating extended defect present in this material. 
Fig. 5. Density of threading dislocations plotted vs. distance from the substrate. A point marked by a crossed square represents published data obtained for a very thick HVPE GaN layer (see Ref. 35). Solid line represents the best fit of the equation (1) to the experimental points (a value of $308 \pm 35 \AA$ for the K parameter was obtained).

Fig. 6. A pair of bright-field TEM images of the $2.5 \mu \mathrm{m}$-thick layer recorded under twobeam conditions for the g-vector: (a) perpendicular and (b) parallel to the c-axis. Notice that the numbers of all types of dislocations are similar.

Fig. 7. A pair of dark-field TEM images of the $5.5 \mu \mathrm{m}$-thick layer recorded under twobeam conditions for the g-vector: (a) perpendicular and (b) parallel to the c-axis. Notice that the numbers of edge and screw dislocations are similar, whereas number of mixed dislocations is about two times higher. 


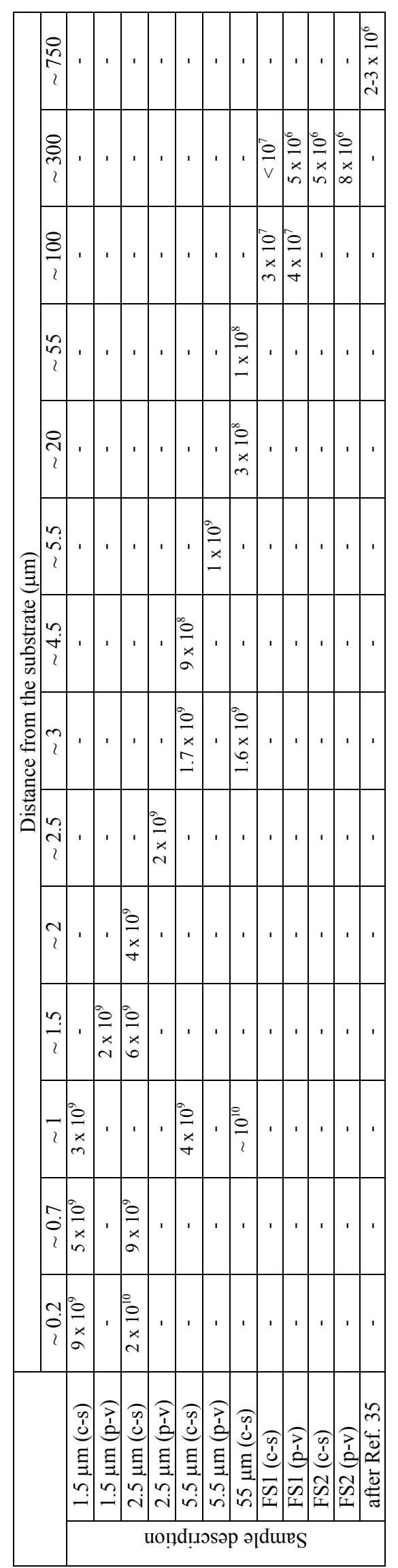

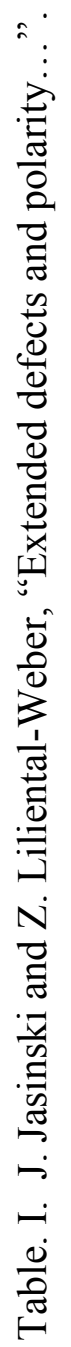




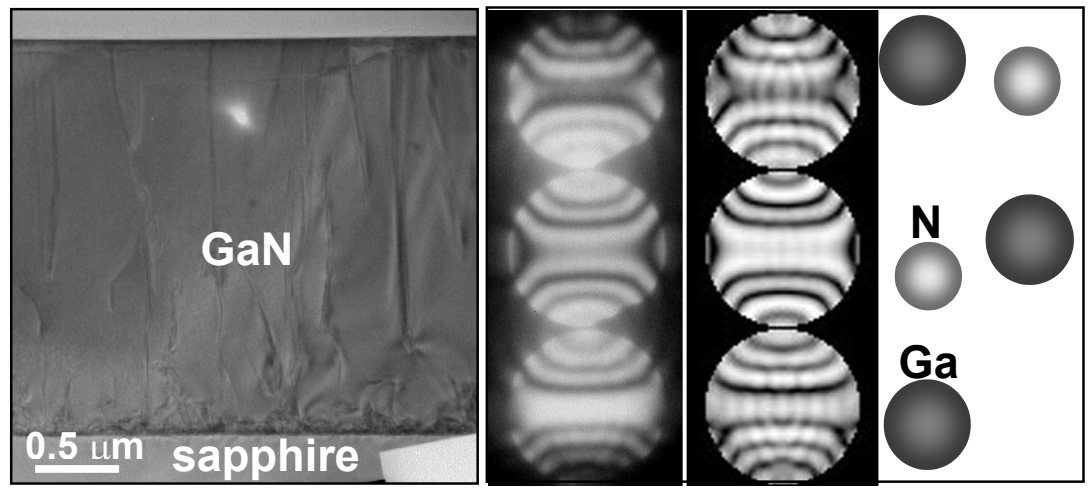

Fig. 1. J. Jasinski and Z. Liliental-Weber, "Extended defects and polarity...". 

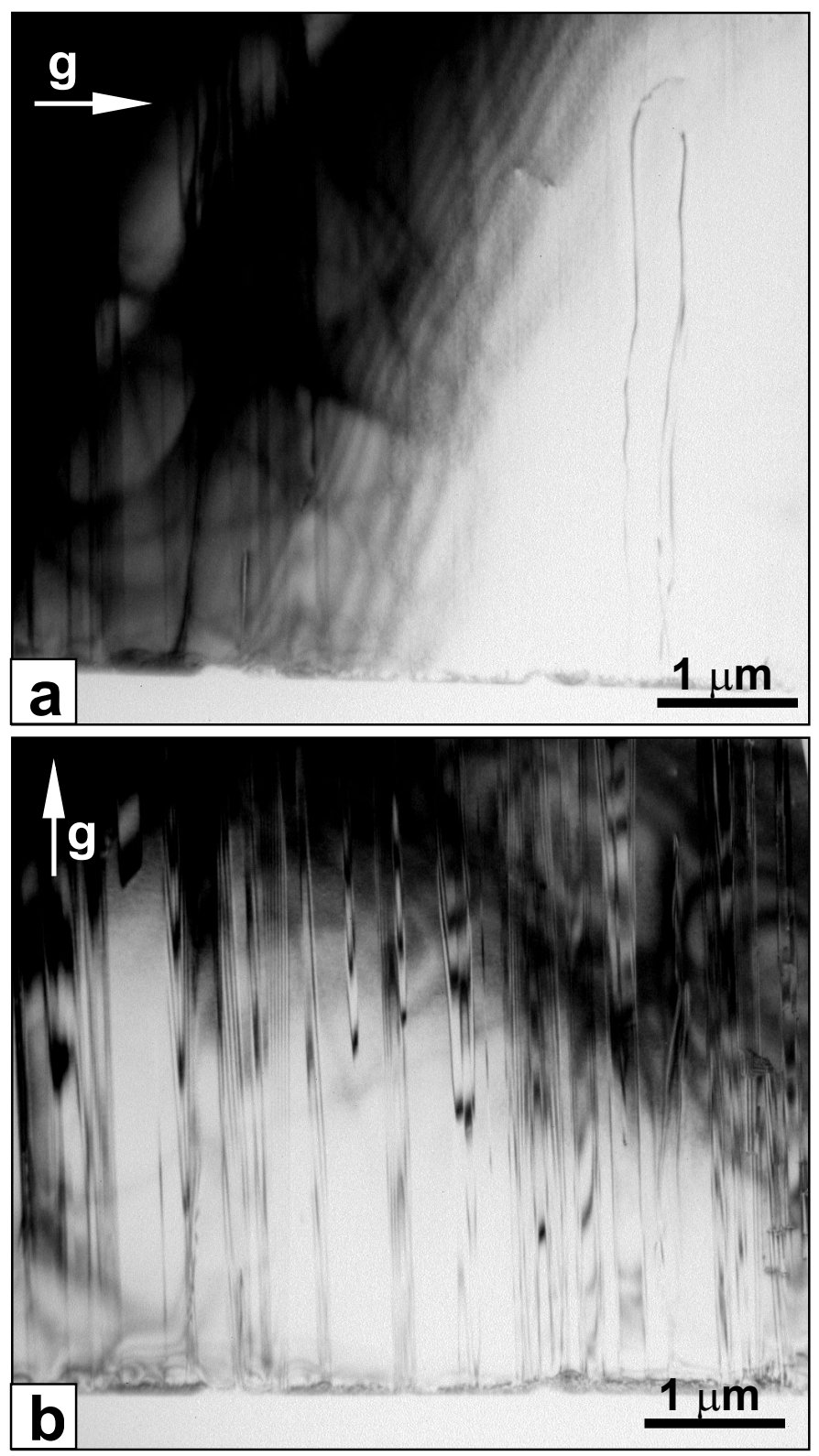

Fig. 2. J. Jasinski and Z. Liliental-Weber, "Extended defects and polarity...". 


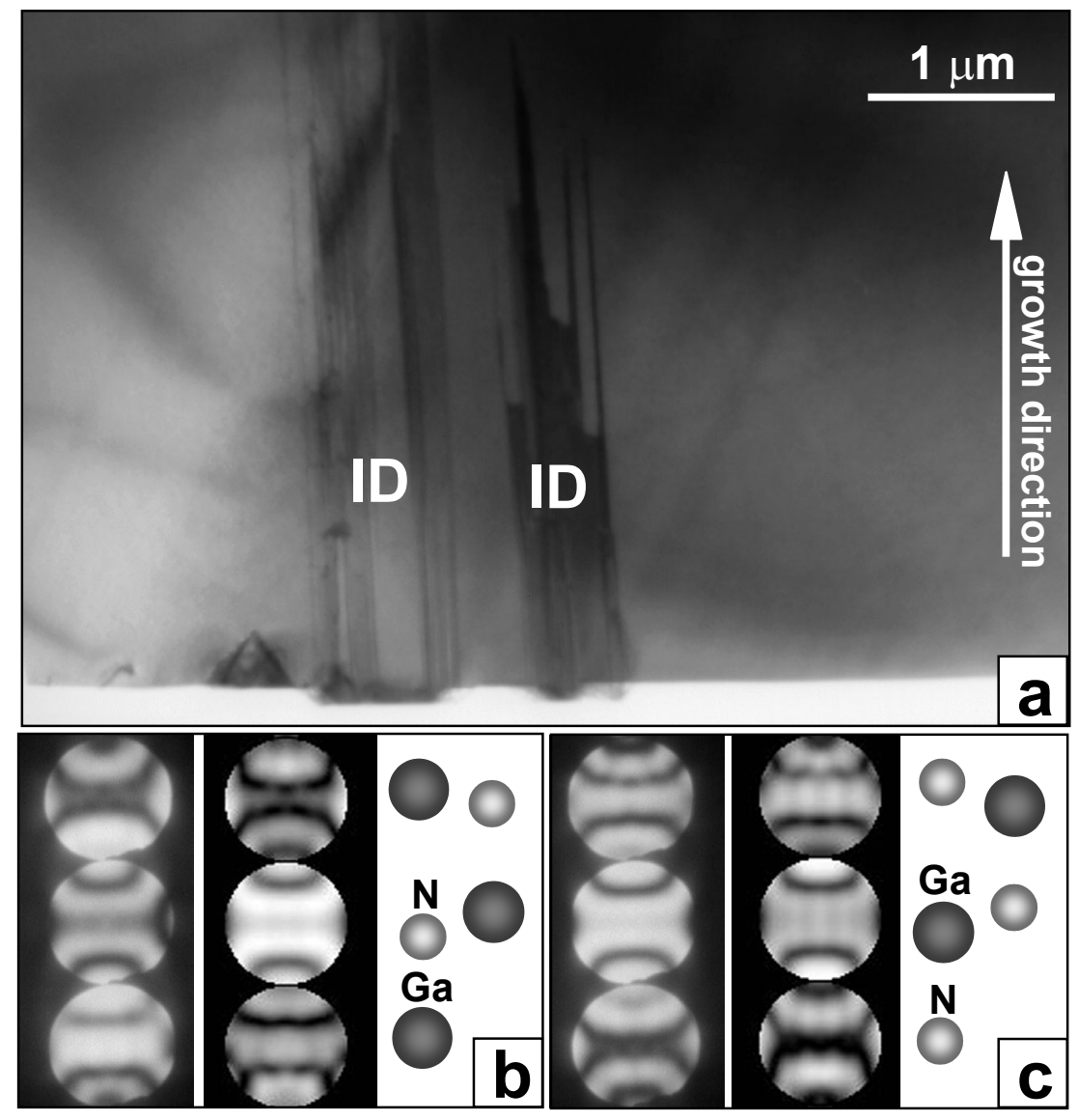

Fig. 3. J. Jasinski and Z. Liliental-Weber, "Extended defects and polarity...”. 

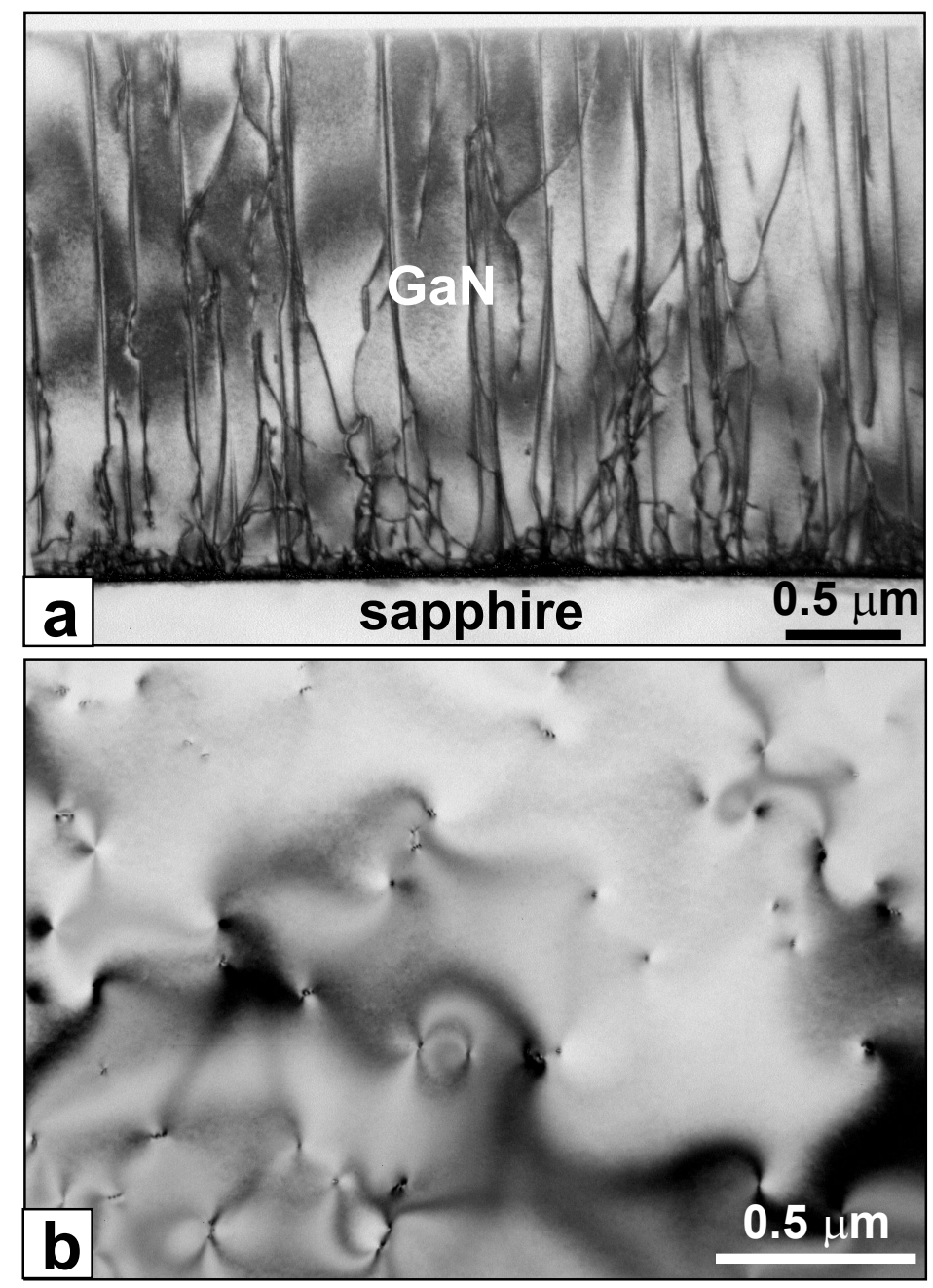

Fig. 4. J. Jasinski and Z. Liliental-Weber, "Extended defects and polarity...". 


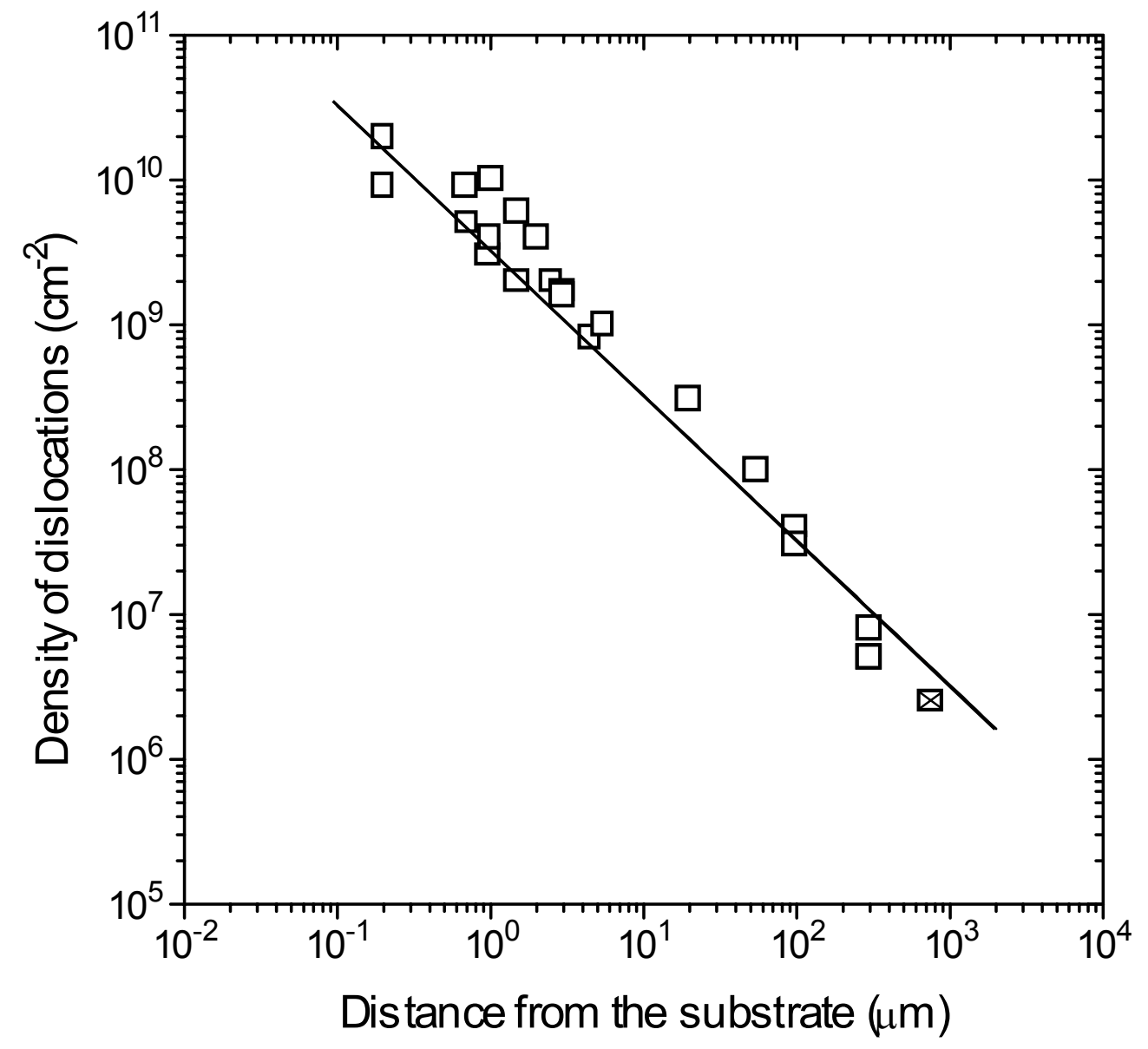

Fig. 5. J. Jasinski and Z. Liliental-Weber, "Extended defects and polarity...". 

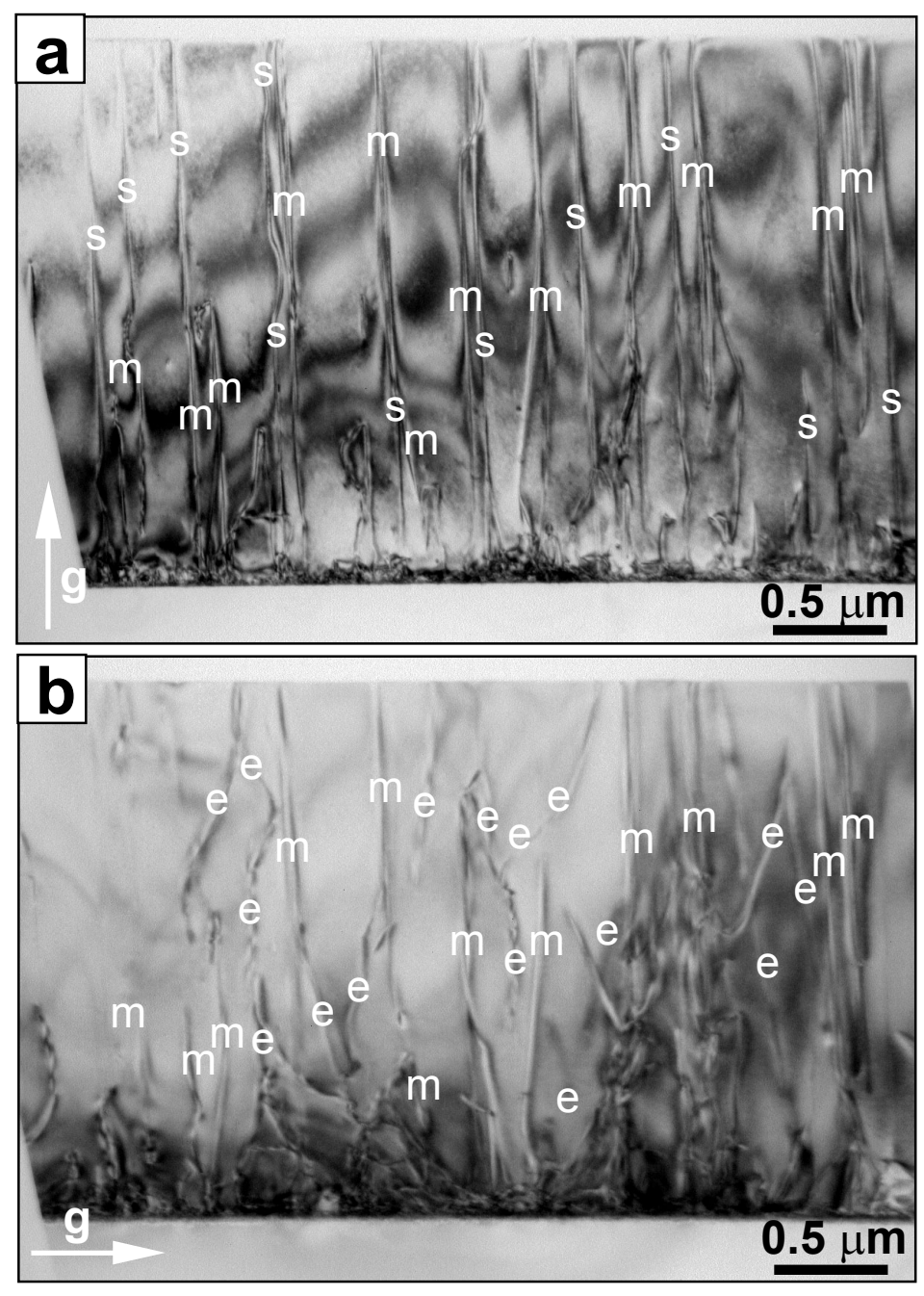

Fig. 6. J. Jasinski and Z. Liliental-Weber, "Extended defects and polarity...". 

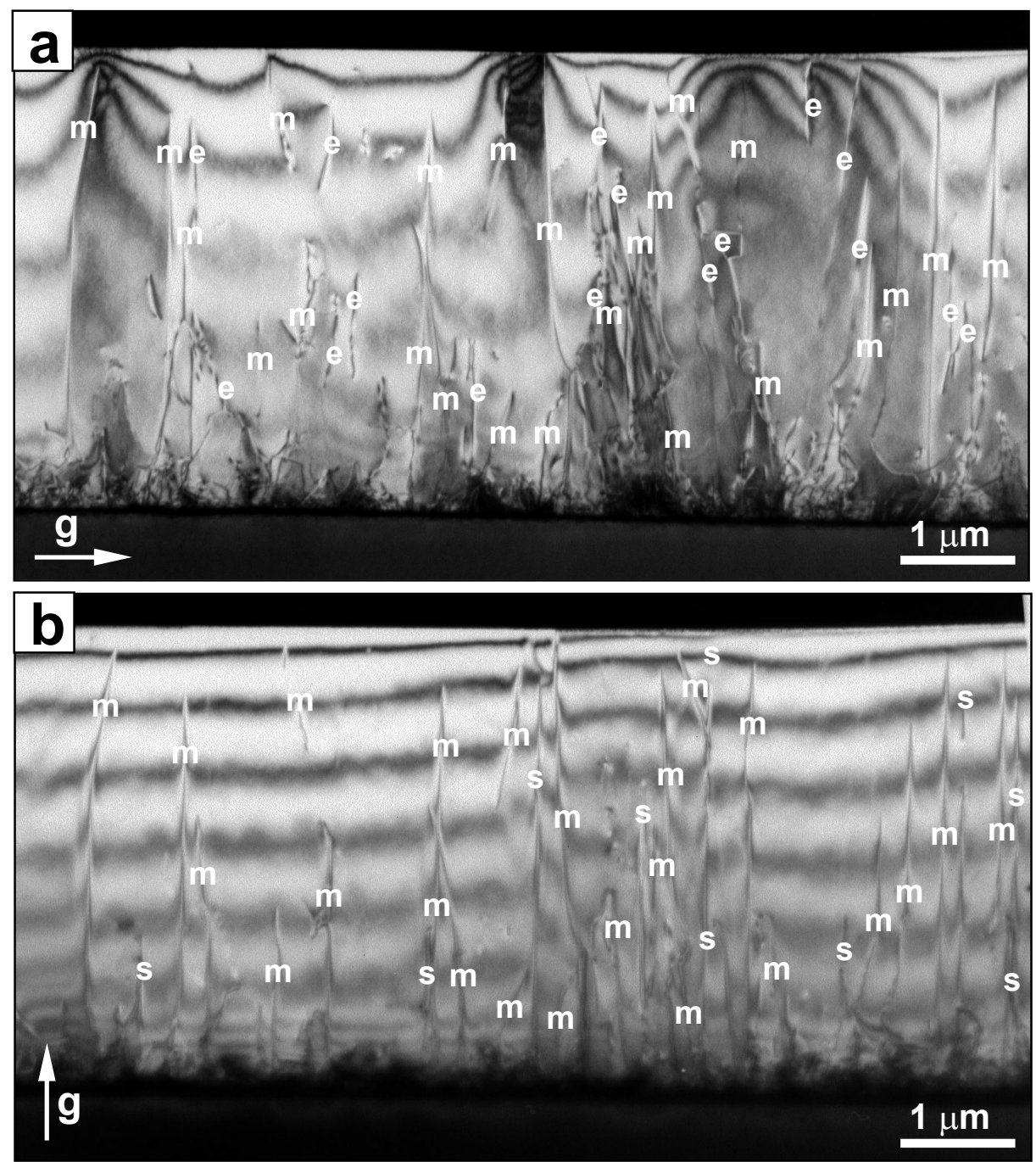

Fig. 7. J. Jasinski and Z. Liliental-Weber, "Extended defects and polarity...". 